\title{
L'Université de Lubumbashi face à la Covid-19 sur les cités universitaires
}

\author{
Par MUMBA KAKUDJI Martial*
}

\begin{abstract}
Résumé :
La pandémie Covid-19 a bouleversé le monde entier et inquiété toute la Communauté internationale. L'Etat congolais, comme tant d'autres, avait fermé temporairement les institutions d'enseignement primaire, secondaire, supérieur et universitaire en vue de bien gérer la crise et limiter les possibilités de propagation du virus. Après le déconfinement, la République Démocratique du Congo, en sigle RDC, a ouvert ces institutions, plus particulièrement universitaires, mais tout en restant ferme aux mesures barrières et instructions édictées par l'Organisation mondiale de la santé et le Ministère de la santé. Cette réflexion aborde la question de la mise en ouvre de ces instructions et mesures barrières dans les cités universitaires de l'Université de Lubumbashi, la gestion de la crise sanitaire et les défis à relever.
\end{abstract}

\section{Introduction}

La pandémie Covid-19, dont le virus responsable est le Coronavirus 2019 autrement appelé 2019-nCoV, identifié pour la première fois à Wuhan, dans la province du Hubei, en Chine, après s'être rependue dans tous les cinq continents, a bouleversé le monde entier et inquiété toute la Communauté internationale. Tous les Etats étaient obligés de prendre les stratégies pouvant réduire les possibilités de la propagation du virus responsable de la pandémie. C'est dans cette perspective que l'Etat congolais, à l'instar d'autres Etats, avait fermé temporairement plusieurs services qui présentaient des grandes possibilités de contaminations, c'est notamment : le secteur de tourisme ${ }^{1}$, le secteur éducatif (ou d'enseignement), voire les autres activités privées telles que les commerces, les rassemblements populaires comme festivals, religieux etc.

* Chef de Travaux, Doctorant à la Faculté de Droit de l'Université de Lubumbashi et Avocat au Barreau du Haut-Katanga, adresse e-mail : martialkakudji@yahoo.fr.

1 Ce secteur est l'un des secteurs les plus durement touchés par la pandémie de Covid-19 et aucun pays n'a été épargné. En effet, les restrictions sur les voyages et la chute soudaine de la demande des consommateurs ayant entrainé une chute sans précèdent du nombre de touristes internationaux. Conformément aux données de l'Organisation mondiale de tourisme (OMT), on note que pas moins de 100 millions d'emplois directs dans le secteur du tourisme sont menacés et que la chute massive des recettes d'exploitation du tourisme pourrait réduire le PIB mondial jusqu'à $2,8 \%$. Permalien : https://www.unwto.org/fr/news/secretaire-general-de-1-onu-il-est-imperatif-que-nous-constuisons-le -secteur-du-tourisme consulté le 10 décembre 2020. Surtout que les voyageurs non contrôlés étaient considéré comme présentant un risque. 
En particulier, le secteur d'enseignement, présentait le grand risque de contamination d'autant plus qu'il met en contact les étudiants ou les élèves, ainsi, vu le mode de transmission dudit virus, s'il y a une personne atteinte dans des institutions plus peuplées où c'est difficile d'observer les mesures de distanciation, cela pouvait véhiculer plus rapidement le virus. C'est pourquoi sous d'autres cieux, les cours étaient dispensés par visioconférence. Mais en République Démocratique du Congo, en sigle RDC, et bien d'autres pays en développement, ce mode de dispensation des cours à distance ne pouvait pas tenir, pour de raisons suivantes : non seulement qu'il y a manque d'équipements nécessaires pour dispenser efficacement l'enseignement à distance; aussi la plupart des enseignants n'avaient pas les compétences pour y parvenir ${ }^{2}$ et leurs enseignés aussi, ne possédaient pas tous les matériels (téléphone intelligent ou ordinaire portable) pouvant permettre ce mode d'apprentissage.

C'est ainsi que, la sécurité des enseignants et enseignés étant primordiale, les institutions d'enseignement (primaire, secondaire, supérieur et universitaire) ont été fermées temporairement en RDC, en vue de bien gérer la crise et limiter les possibilités de la propagation du virus. Après déconfinement, l'Etat congolais a ouvert les institutions d'enseignement, plus particulièrement universitaire, mais tout en restant ferme aux mesures barrières et les instructions édictées par l'Organisation mondiale de la santé, en sigle OMS et le Ministère national de la santé.

Cette réflexion aborde la question de la mise en œuvre de ces instructions et mesures barrières dans les cités universitaires de l'Université de Lubumbashi, la gestion de la crise sanitaire par l'institution et les défis à relever. Elle comporte trois points essentiels : d'abord, nous faisons une description de l'avènement du Coronavirus et la fermeture des institutions d'enseignement en RDC (A); ensuite, nous étudions la situation de la réouverture des institutions d'enseignement universitaire et la mise en œuvre des mesures barrières dans les cités universitaires de l'Université de Lubumbashi (B) et enfin, nous décelons certains défís qui restent à relever $(\mathrm{C})$.

\section{A. l'avènement du coronavirus et la fermeture des institutions d'enseignement en République Démocratique du Congo}

Avant d'aller plus loin, nous devons dire un mot sur le virus responsable de Covid-19 (I) et après parler de circonstance de fermeture des écoles et les universités en RDC (II).

\section{Notions sur la Covid-19}

La Covid-19 est une maladie respiratoire aiguë causée par le virus responsable appelé Coronavirus, qui touche principalement les poumons. Comme nous l'avons déjà dit, la maladie 
tire son origine à Wuhan, dans la province du Hubei, en Chine où le virus responsable était identifié pour la première fois.

Son mode de transmission, selon l'OMS et ce, sur la base des données scientifiques disponibles en mars 2020, le virus de la COVID-19 se transmet d'une personne à une autre par l'intermédiaire des gouttelettes, des fomites et des contacts étroits, avec une propagation possible par les selles. Sa transmission ne se fait pas par voie aéroportée ${ }^{3}$. En avril ces données furent actualiser, d'après les informations récoltées en avril 2020, selon l'OMS, il semblait que le virus de la COVID-19 se transmettait principalement de deux manières : par les gouttelettes respiratoires et par contact ${ }^{4}$.

Des gouttelettes respiratoires sont expulsées par les sujets infectés qui toussent ou qui éternuent. Toute personne en contact étroit (à moins de $1 \mathrm{~m}$ ) avec quelqu'un qui présente des symptômes respiratoires (toux, éternuements) risque d'être exposée à des gouttelettes respiratoires potentiellement infectieuses. Ces gouttelettes peuvent aussi se retrouver sur des surfaces où le virus pourrait rester viable. L'environnement immédiat d'un sujet infecté peut donc être une source de transmission (par contact) ${ }^{5}$.

L'OMS a en avril fait la synthèse de rapports sur la transmission du virus de la COVID-19 et a donné un bref aperçu des données actuelles sur la transmission de ce dernier par des sujets infectés symptomatiques, présymptomatiques et asymptomatiques ${ }^{6}$.

A ce jour, selon l'OMS, les symptômes suivants peuvent être notés : fièvres, la toux sèche, la fatigue, glaires, le souffle court, douleurs musculaires ou articulaires, mal de gorge, le mal de tête, les frissons, les vomissements, le nez bouchés, la diarrhée ${ }^{7}$.

\section{Circonstance de fermeture des écoles et les universités en RDC}

La maladie lorsqu'elle a commencé à faire beaucoup de morts dans les pays occidentaux, chaque Etat avait trouvé judicieux de prendre des mesures barrières pour éviter que la maladie pénètre ses frontières, ou soit dans le cas où elle est déjà atterrie (comme c'est fut le cas en RDC), pour identifier les personnes atteintes et poursuivre avec leur prise en charge.

La RDC n'étant pas épargnée, c'est fut le mercredi 18 mars 2020 que le Présidant de la République, Felix Antoine Tshisekedi Tshilombo, avait pris les mesures consécutivement

3 OMS, Conduite à tenir en matière de lutte anti-infectieuse pour la prise en charge sécurisée du corps d'une personne décédée dans le contexte de la COVID-19, Orientations provisoires, 24 mars 2020 , p. 1.

4 OMS, Conseils sur le port du masque dans le cadre de la COVID-19, Orientations provisoires, 6 avril 2020, p.1.

5 Water, sanitation, hygiene and waste management for COVID-19 https://www.who.int/publicationsd etail/water-sanitation-hygiene-and-wastemanagement-for-covid-19. Cité par ibid.

6 Un cas asymptomatique confirmé en laboratoire correspond à un sujet infecté par le virus de la COVID-19 qui ne présente pas de symptômes. La transmission asymptomatique est la transmission du virus par un sujet qui ne présente pas de symptômes. Ibid.

7 OMS, Coronavirus, Pour se protéger et protéger les autres, affiche. 
avec la décision d'état d'urgence sanitaire, pour endiguer la propagation de la pandémie du Coronavirus dans le pays. La plus grande raison de la prise de ces mesures était que : Sept nouveaux cas de Coronavirus ont été notifiés à Kinshasa, ce mercredi-là, en une journée, le nombre de cas était passé de sept à quatorze ${ }^{8}$.

Le contenu de mesures qui étaient prises par le Chef de l'Etat pour endiguer la propagation du Coronavirus et la prise en charge des personnes infectées avait deux points focaux, à savoir : l'accès au territoire national et les rassemblements populaires ${ }^{9}$.

En ce qui concerne l'accès au territoire national :

- la suspension, jusqu'à nouvel ordre dès le vendredi 20 mars 2020, de tous les vols en provenance des pays à risque et des pays de transit. Seuls les avions et les navires cargos et autres moyens de transport frets étaient autorisés à accéder au territoire national et leurs personnels soumis aux contrôles;

- report des voyages à destination de la République Démocratique du Congo de tous les passagers résidant dans les pays à risque jusqu'à nouvel ordre;

- obligation à tous les passagers, à leur arrivée aux frontières nationales, de remplir une fiche de renseignements et de se soumettre, sans exception, à l'obligation de lavage des mains et du prélèvement de la température;

- obligation de mise en quarantaine de 14 jours maximum à toute personne suspectée à l'issue du test de température, pour un examen approfondi et au besoin d'interner, dans les hôpitaux prévus à cet effet, les personnes qui seront testées positives;

- de doter tous les postes d'entrée maritime, fluviale, lacustre et terrestre du territoire national du même dispositif de surveillance pour renforcer le contrôle des passagers en provenance de l'étranger;

- et en fin, de soumettre systématiquement les personnes en partance de Kinshasa vers les différentes provinces de la RDC aux mesures de contrôle dans le but d'éviter la propagation de cette pandémie sur le reste de la $R D C$.

En ce qui concerne les rassemblements populaires :

- l'interdiction de tous rassemblements, réunion, célébrations, de plus de 20 personnes sur les lieux publics en dehors du domicile familial;

- fermeture des écoles, des universités, des instituts supérieurs officiels et privés sur l'ensemble du territoire national à compter du jeudi 19 mars 2020 (soit un jour après mesures) pour une durée de 4 semaines;

- suspension de tous les cultes pour une période de 4 semaines à compter du jeudi 19 mars 2020;

- suspension des activités sportives dans les stades et autres lieux de regroupement sportif jusqu'à nouvel ordre;

8 BBC NEWS, «Coronavirus : le président de la RDC, Felix Tshisekedi prend des mesures drastiques ", publié le 19 mars 2020 et consulté le 11 décembre 2020 sur le Permalien : https://www.bbc .com/afrique/region-51959819.

9 Ibid. 
- interdiction, toujours jusqu'à nouvel ordre, de l'ouverture des discothèques, bars, cafés, terrasses et restaurants;

- interdiction de l'organisation des deuils dans les salles et les domiciles. Les dépouilles mortelles devraient être conduites directement de la morgue jusqu'au lieu d'inhumation et en nombre restreint d'accompagnateurs;

- et enfin, prise en charge aux frais du Gouvernement tous les cas testés positifs sur l'ensemble du territoire.

Comme nous le constatons, il ressort de ces mesures que le secteur éducatif ou mieux de l'enseignement, entrant dans ce qu'on appelle les rassemblements populaires, fut touché par les mesures prises dans le cadre de riposte contre la pandémie. Et pourtant, conformément au programme national, les institutions universitaires plus particulièrement l'Université de Lubumbashi venait de clôturer le premier semestre.

En effet, la fermeture des écoles, des universités, des instituts supérieurs officiels et privés sur l'ensemble du territoire national, conformément à la décision précitée, avait débuté le jeudi 19 mars 2020 (soit un jour après mesures) pour une durée de 4 semaines. Cette décision devrait prendre fin le Jeudi le 16 avril 2020. Cependant, en date du 24 mars (soit 6 jours après la prise des mesures), l'état d'urgence fut décrété, tout en isolant la Ville province de Kinshasa des autres provinces du pays ${ }^{10}$. Cela avait pour conséquence, la date référence devrait être celle de l'état d'urgence, qui devrait prendre fin, conformément à l'alinéa 3 de l'article 144 de la Constitution ${ }^{11}$, le 24 avril 2020.

Apres l'épuisement du délai de 30 jours, l'ordonnance proclamant l'état d'urgence devrait être prorogée car l'article précité de la Constitution prévoit que «l'ordonnance proclamant l'état d'urgence ou l'état de siège cesse de plein droit de produire ses effets après l'expiration du délai prévu à l'alinéa trois du présent article, à moins que l'Assemblée nationale et le Sénat, saisis par le Président de la République sur décision du Conseil des ministres, n'en aient autorisé la prorogation pour des périodes successives de quinze jours $»^{12}$. C'est ainsi que le Parlement de la RDC avait sur demande du Président de la

10 C'est fut mardi que le Présidant de la République avait décrété l'état d'urgence ainsi que l'isolement de la capitale Kinshasa en ces termes : "je décrète l'état d'urgence, l'interdiction de tous les voyages de Kinshasa vers les provinces, et des provinces vers Kinsahsa, afin de permettre le confinement de la ville de Kinshasa, foyer de la pandémie ». Belga, "Coronavirus : la RDC décrète "l'état d'urgence" et isole Kinshasa du reste du pays ", in RFBF.Be, Publié le 24 mars 2020. A trouver en ligne sur : https://www.rtbf.be/info/monde/detail-coronavirus-la-rdc-decrete-l-e tat-d-urgence-et-isole-kinshasa-du-reste-du-pays?id=10466723.

11 Cet alinéa ce qui suit : "L'état d'urgence ou l'état de siège peut être proclamé sur tout ou partie du territoire de la République pour une durée de trente jours». Article 144 de la Loi no 11/002 du 20 janvier 2011 portant révision de certains articles de la Constitution de la République Démocratique du Congo du 18 février 2006, JORDC, Numéro Spécial 52ème Année, Kinshasa - 5 février 2011.

12 Ibid. 
République, voté la prorogation de 15 jours de l'état d'urgence ${ }^{13}$. Cette situation a perduré quatre mois successifs et les institutions universitaires étaient toujours fermées dans le pays.

Le 21 juillet 2020, le Chef de l'Etat avait levé la mesure d'état d'urgence, assortie d'un calendrier de reprise progressive des activités dans le pays, y compris la réouverture des écoles, universités et autres établissements d'enseignement. C'est ce que le point suivant analyse en détail et les mesures barrières qui devraient être observées.

\section{B. la réouverture des institutions d'enseignement universitaire et la mise en œuvre des mesures barrières dans les cités universitaires de l'Université de Lubumbashi}

En réalité, la levée des mesures de fermeture des activités en RDC en date du 21 juillet 2020, n'avait pas autorisé l'ouverture immédiate des institutions d'enseignement. La décision prévoyait que : "les écoles, universités et autres établissements d'enseignements ouvriront le 3 aout 2020 ...» ${ }^{14}$. Mais, les exigences de distanciation sociale et le port de masques en public devraient se poursuivre.

Les mesures barrières bien que chaque Etat, au travers de son ministère de santé publique s'en a appropriées, mais celles-ci proviennent des organisations internationales chargées des questions de santé publique, dont en premier lieu, on voit l'OMS ${ }^{15}$. Cette dernière a été mise en place par les Etats dans le but de coopérer entre eux et avec tous autres pour améliorer et protéger la santé de tous les peuples et aux termes de l'exposé des motifs de sa Constitution, l'Organisation mondiale de la Santé est une institution spécialisée aux termes de l'article 57 de la Charte des Nations Unies, qui en vertu de son Chapitre Ier, a pour but «d'amener tous les peuples au niveau de santé le plus élevé possible ${ }^{16}$.

Par ailleurs, les mesures et instructions devant provenir d'elle, face aux urgences sanitaires qui ont affecté sérieusement toutes les Nations de l'Organisation des Nations Unies, cette organisation avait en date du 26 janvier 2020 élaboré les listes de contrôle en matière de communication sur les risques et de participation communautaire (RCCE) pour la préparation et les premières ripostes face au nouveau coronavirus 2019 (2019-nCoV).

13 Belga, «Coronavirus en RDC : le Parlement vote la prorogation de 15 jours de l'état d'urgence », in RFBF.Be, Publié le 23 avril 2020. A trouver en ligne sur : https://www.rtbf.be/info/monde/detail -coronavirus-en-rdc-le-parlement-vote-la-prorogation-de-15-jours-de-l'état-d-urgence?id=1048843 4.

14 https://www.bbc.com/afrique/region-53497849 Consulté le 12 décembre 2020.

15 La Constitution de l'OMS

Voir la Constitution de l'Organisation mondiale de la santé, adoptée par la Conférence internationale de la Santé, tenue à New York du 19 juin au 22 juillet 1946, entrée en vigueur le 7 avril 1948 et amendée par la Vingt-Sixième, la Vingt-Neuvième, la Trente-Neuvième et la Cinquante et Unième Assemblée mondiale de la Santé (résolutions WHA26.37, WHA29.38, WHA39.6 et WHA51.23) entrés en vigueur le 3 février 1977, le 20 janvier 1984, le 11 juillet 1994 et le 15 septembre 2005 respectivement.

16 Article $1^{\text {er }}$ de la Constitution de l'OMS. 
Dans ce document on trouve des conseils pratiques que l'organisation donne aux pays pour la mise en œuvre de stratégies efficaces en matière de RCCE qui contribueront à protéger la santé publique lors des premières phases de la riposte face à unCoV ${ }^{17}$.

A la lumière de cette communication, l'organisation avait proposé trois listes : la première liste est relative au contrôle de l'état de préparation à la RCCE à l'intention des pays se préparant à un éventuel cas importé de 2019-nCoV (aucun cas n'ayant encore été identifié); la deuxième relative au contrôle de la première riposte en matière de RCCE à l'intention des pays où un ou plusieurs cas de 2019-nCoV ont été identifiés; la troisième liste enfin, relative au contrôle pour la RCCE en cas de crise à l'intention des pays où la transmission du 2019-nCoV est en cours ${ }^{18}$.

Dans le contexte de l'Université de Lubumbashi, vu que la RDC était déjà parmi les Etats qui avaient plusieurs cas de Covid-19, pour la reprise des cours en toute sécurité, les mesures ci-après devraient être mises en œuvre.

\section{Mesures à prendre proposées par l'OMS}

Ces mesures ont été proposées par l'OMS au Gouvernement de l'Etat qui a un ou plusieurs cas de Covid-19 déjà identifiés. Elles portent notamment sur : Systèmes de communication sur les risques (1); Coordination interne et entre partenaires (2); Communication publique (3); Participation communautaire (4); Tenir compte des incertitudes et des différentes perceptions, et gérer les mauvaises informations (5) et Renforcement des capacités (6).

1. Systèmes de communication sur les risques

Pour tout Etat concerné, il faut :

- Adapter le plan de RCCE existant à la riposte, faire entrer en action l'équipe d'intervention et déclencher le plan de RCCE;

- Faire intervenir le porte-parole désigné pour la situation d'urgence;

- Établir un calendrier pour les activités et les produits de communication;

- Assurer le suivi de la riposte en matière de RCCE en identifiant les processus qui retardent la diffusion des informations et sèment la confusion chez les populations touchées.

17 OMS, Communication sur les risques et participation communautaire (RCCE) : préparation et riposte face au nouveau coronavirus 2019 (2019-nCoV), Lignes directrices provisoires, 26 janvier 2020, p. 1.

18 OMS, Communication sur les risques et participation communautaire (RCCE), op cit., pp. 3-8. 
2. Coordination interne et entre partenaires

Sur ce point, il faut :

- actionner les modes opératoires normalisés (MON) afin de coordonner les activités de RCCE avec celles d'autres organismes et partenaires impliqués dans la riposte;

- faire le lien entre les opérations de RCCE menées aux niveaux national, régional et local;

- assigner les responsabilités en matière de communication interne (au sein de chaque organisme d'intervention et entre les différents organismes) et de communication externe (à l'intention du public);

- coordonner les phases de préparation, d'harmonisation et de diffusion des messages.

3. Communication publique

Sur ce point, il faut :

- annoncer le premier cas de $n \mathrm{CoV}$ au plus tôt et actualiser les informations après qu'une évaluation des risques et une analyse de la perception du risque ont été entreprises;

- transmettre les informations dès leur réception, même si celles-ci sont incomplètes, et faire part ouvertement du degré d'incertitude (ce qui veut dire gérer l'incertitude); mettre à la disposition du public des canaux de communication traditionnels par lesquels il soit possible d'obtenir des renseignements actualisés (par exemple des permanences téléphoniques, un site Web...);

- élaborer et tester des messages, y compris des messages portant sur des conseils de santé publique;

- s'assurer que les messages soient cohérents entre les différents secteurs et niveaux;

- recourir à des canaux de communication fiables et efficaces que les publics cibles utilisent régulièrement;

- faire participer, former et faire entrer en action des personnes influentes de confiance aux yeux des publics cibles parmi lesquelles, en particulier, des agents de santé.

4. Participation communautaire

Sur ce point, il faut :

- Mener une analyse rapide de la perception du risque fondée sur les informations formelles et informelles disponibles;

- assurer un suivi des obstacles possibles à l'adoption de comportements de protection; adapter la communication aux publics cibles (par exemple, personnes touchées, agents de santé, responsables politiques, bailleurs de fonds); traduire la documentation dans les langues pertinentes et l'adapter aux niveaux d'instruction appropriés;

- créer de courtes présentations multimédia présentant les informations clés (qui expliquent par exemple l'étiologie de la maladie, les symptômes, le mode de transmission, 
les moyens de se protéger et comment procéder si quelqu'un tombe malade) susceptibles d'être partagées en ligne et diffusées à la télévision.

5. Tenir compte des incertitudes et des différentes perceptions, et gérer les mauvaises informations

- communiquer sur les éléments connus et inconnus : expliquer le degré d'incertitude qui règne;

- activer des mécanismes de suivi des rumeurs et de riposte à ces rumeurs, et tenter de déterminer les problèmes susceptibles d'en être la cause;

- mettre en place un suivi des médias de masse et des réseaux sociaux, des permanences téléphoniques, des retours obtenus par les agents de santé auprès des patients ainsi que des préoccupations des communautés, et faire remonter continuellement l'information afin d'adapter la stratégie de RCCE.

\section{Renforcement des capacités}

Enfin, en ce qui concerne le renforcement de capacité, il faut :

- projeter de fournir régulièrement des lignes directrices actualisées à tous les acteurs de la RCCE;

- former les personnels de renfort;

- envisager de former les responsables, les acteurs de terrain et les porte-parole aux lignes directrices en matière de RCCE si nécessaire.

Le 1er avril 2020 l'OMS a fait des recommandations aux États Membres pour améliorer les pratiques d'hygiène des mains par un accès universel à des postes publics d'hygiène des mains afin d'aider à prévenir la transmission du virus de la COVID-19. Il ressort de ces recommandations ce qui suit ${ }^{19}$ :

- Un ou plusieurs postes d'hygiène des mains (soit pour se laver les mains au savon et à l'eau, ${ }^{20}$ soit pour se frotter les mains avec une solution hydroalcoolique, ${ }^{21}$ doivent être placés devant l'entrée de chaque bâtiment public (y compris les écoles et les établisse-

19 OMS, Recommandations aux États Membres pour améliorer les pratiques d'hygiène des mains par un accès universel à des postes publics d'hygiène des mains afin d'aider à prévenir la transmission du virus de la COVID-19, Orientations provisoires, 1er avril 2020, pp. 3.

20 En l'absence de solution hydro-alcoolique ou de savon en barre, une solution de savon liquide, mélangeant détergent et eau, peut être utilisée. La proportion de détergent et d'eau dépend du type et de la concentration des produits disponibles localement.

21 Les solutions chlorées pour le lavage des mains ne sont pas recommandées en raison des risques potentiels pour les utilisateurs et les personnes qui préparent ces solutions, ainsi que de la dégradation du chlore exposé à la lumière du soleil ou à la chaleur. Le savon est généralement bon marché et facile à trouver, et on peut également utiliser des solutions de savon liquide. 
ments de santé) ou bâtiment commercial privé, pour permettre à chacun d'appliquer les mesures d'hygiène des mains en entrant et en sortant.

- La quantité et la facilité d'utilisation des postes d'hygiène des mains doivent être adaptées au type (par exemple les jeunes enfants, les personnes âgées, les personnes à mobilité réduite) et au nombre d'utilisateurs afin de mieux encourager leur utilisation et de réduire le temps d'attente.

- L'installation, la supervision et le remplissage régulier des équipements doivent relever de la responsabilité générale des autorités de santé publique et être délégués aux gestionnaires des bâtiments. Les initiatives du secteur privé et de la société civile visant à soutenir les installations, leur entretien et leur utilisation efficace sont les bienvenues.

- L'utilisation de postes publics d'hygiène des mains doit être obligatoire avant de franchir l'entrée de tout bâtiment et de tout moyen de transport public pendant la pandémie de COVID-19.

- Tous les établissements de santé privés et publics doivent établir ou renforcer leurs programmes multimodaux d'amélioration de l'hygiène des mains et assurer rapidement, au minimum, l'achat de quantités suffisantes de fournitures de qualité pour l'hygiène des mains, une formation actualisée sur l'hygiène des mains, ainsi que des rappels et des communications sur l'importance de l'hygiène des mains pour prévenir la propagation du virus de la COVID-19. Tous les établissements de santé sont vivement encouragés à participer activement à la campagne de l'OMS intitulée "Save Lives: Clean Your Hands » avant et le 5 mai 2020 et à répondre à l'Appel mondial à l'action du Secrétaire général des Nations Unies pour l'eau, l'assainissement et l'hygiène (WASH) dans les établissements de santé.

Il y a lieu de saluer l'Université de Lubumbashi qui, par le biais de ses chercheurs, Professeurs Balaka Ekwalanga Michel et Lungu Anzwal Philomène, tous Docteurs en Médicine humaine, ont mis en place dès l'annonce de cette pandémie, un protocole de prise en charge de la Covid19. Ce protocole de prise en charge de la Covid-19 est dénommé : « approche immunothérapeutique contre le Covid -19 Protocome Bella UNILU 20 : combinaison des interférons de type 1 et 2 boostée par la chloroquine et les antioxydants ». Lors de la présentation de ce protocole à la Communauté universitaire, les deux chercheurs ont demandé à cette dernière de mettre en application au jour le jour ce protocole pour éviter la contamination et pour se faire soigner au cas où tout membre de la Communauté serait contaminé. Ceci a été mis en application et aucun cas n'a été détecté jusque-là sur les cités universitaires. Ces chercheurs ont contribué dans la lutte et la prévention contre la Covid-19. Le Gouvernement central a fait appel à eux à Kinshasa où ils ont fait des essais satisfaisants en collaboration avec l'Institut National de Recherche Biomédicale. Pour cela, ils ont bénéficié d'un Brevet d'invention. 
L'OMS a pris aussi des mesures et Conseils sur le port du masque dans le cadre de la COVID-19 dans les milieux publics. De ce texte nous en tirons les pratiques suivies dans les établissements de santé pour un bon usage des masques ${ }^{22}$ ci-dessous :

Les masques, de tout type, doivent absolument être utilisés et éliminés correctement si l'on veut qu'ils soient efficaces et pour éviter d'accroître la transmission. A cet effet, il faut : - placer soigneusement le masque de façon à recouvrir le nez et la bouche et bien serrer les liens pour l'ajuster au mieux sur le visage; • une fois le masque placé, éviter de le toucher; • retirer le masque selon la technique adéquate (ne pas toucher l'avant du masque mais ôter la lanière derrière la tête); • après avoir retiré ou touché par inadvertance un masque usagé, se laver les mains avec une solution hydroalcoolique, ou à l'eau et au savon si elles sont visiblement sales; - s'il est humide, remplacer le masque par un nouveau masque propre et sec; • ne pas réutiliser les masques à usage unique; • jeter les masques à usage unique après chaque utilisation et immédiatement après les avoir retirés.

En tout état de cause, ces orientations sont provisoires selon l'OMS. C'est-à-dire qu'elle continue à suivre de près la situation et reste attentive à tout changement susceptible d'avoir une incidence sur ces orientations. Mais ces orientations, peu importe de nouvelle mise à jour en cas de changements, l'OMS estime que, ce document d'orientation provisoire expirera deux ans après la date de publication ${ }^{23}$.

Outre ces mesures, il y a des mesures prises toujours par l'OMS et proposées comme recommandations aux Etats pour des cas particuliers dans la gestion de la pandémie. On peut citer entre autres : les mesures relatives à la conduite à tenir en matière de lutte anti-infectieuse pour la prise en charge sécurisée du corps d'une personne décédée dans le contexte de la COVID-1924; celles relatives à la protection de soi et des autres ${ }^{25}$ etc.

\section{C. les défis qui restent à relever}

La gestion de la Covid-19 dans les cités universitaires de l'Université de Lubumbashi, outre les mérites et les efforts relevés ci-haut, a cependant des faiblesses qui convient de relever et proposer des pistes de solutions.

22 OMS, Conseils sur le port du masque dans le cadre de la COVID-19, op cit., p. 4.

23 OMS, Conseils sur le port du masque dans le cadre de la COVID-19, op cit, p. 4.

24 OMS, Conduite à tenir en matière de lutte anti-infectieuse pour la prise en charge sécurisée du corps d'une personne décédée dans le contexte de la COVID-19, Orientations provisoires, 24 mars 2020, pp. 6.

25 L'OMS propose pour se protéger et protéger les autres les mesures suivantes : « se laver régulièrement les mains avec l'eau et du savon; Tousser ou éternuer dans son coude ou dans un mouchoir; Utiliser un mouchoir jetable; Eviter tout contact corporel inutile; Port de masque chirurgical jetable lorsque l'on est malade ». Voir OMS, Coronavirus, Pour se protéger et protéger les autres, affiche. 
Il sied de le mentionner, pour se protéger et protéger les autres, c'est d'abord la question de conscience individuelle de tout un chacun. La culture, l'éducation, et religion peuvent aussi influer sur l'attitude de tout un chacun.

Malgré le risque que présente le virus responsable de la maladie de Covid-19 et les possibilités multiples de la propagation rapide, force est de constater que, certains étudiants demeurent inciviques. Pour certains, ils portent de masques mais pas comme il est recommandé de le faire (supra). Certains les portent en dessous du nez, les autres n'en portent même pas, ou ils n'en portent que lorsqu'ils trouvent que c'est exigible.

Certains malgré la mise en place des postes publics d'hygiène des mains afin d'aider à prévenir la transmission du virus de la COVID-19, tels que recommandés par l'OMS aux Etats membres, ils ne se lavent même pas les mains.

Toutes ces déviations proviennent soit de certaines rumeurs et mauvaises informations sur la maladie. Pour certains, la Covid-19 n'existe pas, pour certains autres, c'est une maladie qui tuent seulement les blancs et non les personnes noires (pour dire les africains). Il y a même d'autres qui croient que Covid-19 est une stratégie qu'utilise les politiques pour effrayer la population, les confiner ou c'est une maladie qui tue uniquement les païens (entendu comme non chrétiens) etc.

C'est sur base de ces remarques que nous proposons aux étudiants, voire les enseignants d'être consciencieux et de bien vouloir aider le gouvernement de la RDC, plus particulièrement, le ministère de santé de la RDC, les autorités provinciales, et le Comité de gestion de l'Université de Lubumbashi de mettre en œuvre les recommandations de l'autorité mondiale de santé publique, en vue de combattre ensemble la pandémie de Covid-19 dans les milieux universitaires.

\section{Conclusion}

Pour conclure, il est certain qu'après l'ouverture des institutions d'enseignements, pour éviter la propagation du virus, le gouvernement congolais était resté ferme aux mesures barrières et les instructions édictées par l'Organisation mondiale de la santé et le Ministère de la santé. Nos investigations, ont révélé que dans les cités universitaires de l'Université de Lubumbashi, celle-ci a mis en place les dispositifs tels que recommandés par l'OMS et le Ministère de santé de la RDC, afin de lutter contre la propagation du virus. En plus, il est exigé aux étudiants et les enseignants de porter les masques, et respecter toutes les mesures barrières.

Cependant, on constate parfois des résistances telles que : la non-observation de la mesure de port obligatoire des masques; le port des masques mais de manière non recommandée.

Force est de mentionner aussi les efforts fournis par l'Université de Lubumbashi qui, par le biais de ses chercheurs, Docteurs en Médicine humaine, ont mis en place dès l'annonce de cette pandémie, un protocole dénommé : «approche immunothérapeutique 
contre le Covid-19 Protocome Bella - UNILU 20 : combinaison des interférons de type 1 et 2 boostée par la chloroquine et les antioxydants », pour la prise en charge de la Covid19.

\section{Bibliographie sélective}

1. $B B C$ NEWS, «Coronavirus : le président de la RDC, Felix Tshisekedi prend des mesures drastiques », publié le 19 mars 2020 et consulté le 11 décembre 2020 sur le Permalien : https://www.bbc.com/afrique/region-51959819

2. Belga, «Coronavirus en RDC : le Parlement vote la prorogation de 15 jours de l'état d'urgence », in RFBF.Be, Publié le 23 avril 2020. A trouver en ligne sur : https://www .rtbf.be/info/monde/detail-coronavirus-en-rdc-le-parlement-vote-la-prorogation-de-15 -jours-de-l'état-d-urgence?id=10488434

3. Belga, «Coronavirus : la RDC décrète "l'état d'urgence" et isole Kinshasa du reste du pays », in RFBF.Be, Publié le 24 mars 2020. A trouver en ligne sur : https://www.r tbf.be/info/monde/detail-coronavirus-la-rdc-decrete-l-etat-d-urgence-et-isole-kinshasa -du-reste-du-pays?id=10466723

4. Constitution de l'Organisation mondiale de la santé, adoptée par la Conférence internationale de la Santé, tenue à New York du 19 juin au 22 juillet 1946, entrée en vigueur le 7 avril 1948 et amendée par la Vingt-Sixième, la Vingt-Neuvième, la Trente-Neuvième et la Cinquante et Unième Assemblée mondiale de la Santé (résolutions WHA26.37, WHA29.38, WHA39.6 et WHA51.23) entrés en vigueur le 3 février 1977, le 20 janvier 1984, le 11 juillet 1994 et le 15 septembre 2005 respectivement.

5. https://www.bbc.com/afrique/region-53497849

6. https://www.ilo.org/sector/Resources/publications/WCMS-745392/lang--fr/index.htm

7. https://www.unwto.org/fr/news/secretaire-general-de-1-onu-il-est-imperatif-que-nous -constuisons-le-secteur-du-tourisme

8. Loi $\mathrm{n}^{\circ} 11 / 002$ du 20 janvier 2011 portant révision de certains articles de la Constitution de la République Démocratique du Congo du 18 février 2006, JORDC, Numéro Spécial 52ème Année, Kinshasa - 5 février 2011.

9. OMS, Communication sur les risques et participation communautaire (RCCE) : préparation et riposte face au nouveau coronavirus 2019 (2019-nCoV), Lignes directrices provisoires, 26 janvier 2020

10. OMS, Conduite à tenir en matière de lutte anti-infectieuse pour la prise en charge sécurisée du corps d'une personne décédée dans le contexte de la COVID-19, Orientations provisoires, 24 mars 2020.

11. OMS, Conduite à tenir en matière de lutte anti-infectieuse pour la prise en charge sécurisée du corps d'une personne décédée dans le contexte de la COVID-19, Orientations provisoires, 24 mars 2020, pp. 6.

12. OMS, Conseils sur le port du masque dans le cadre de la COVID-19, Orientations provisoires, 6 avril 2020.

13. OMS, Conseils sur le port du masque dans le cadre de la COVID-19, op cit, p. 4. 
14. OMS, Coronavirus, Pour se protéger et protéger les autres, affiche.

15. OMS, Recommandations aux États Membres pour améliorer les pratiques d'hygiène des mains par un accès universel à des postes publics d'hygiène des mains afin d'aider à prévenir la transmission du virus de la COVID-19, Orientations provisoires, 1er avril 2020.

16. Water, sanitation, hygiene and waste management for COVID-19 https://www.who.int /publicationsdetail/water-sanitation-hygiene-and-wastemanagement-for-covid-19. 